\title{
Absolute proper motions of open clusters
}

\section{Observational data ${ }^{\star}$}

\author{
H. Baumgardt ${ }^{1}$, C. Dettbarn ${ }^{2}$, and R. Wielen ${ }^{2}$ \\ 1 Department of Mathematics and Statistics, University of Edinburgh, Edinburgh EH9 3JZ, UK \\ 2 Astronomisches Rechen-Institut Heidelberg, Mönchhofstraße 12-14, D-69120 Heidelberg, Germany \\ e-mail: holger@maths.ed.ac.uk
}

Received May 29; accepted July 17, 2000

\begin{abstract}
Mean proper motions and parallaxes of 205 open clusters were determined from their member stars found in the Hipparcos Catalogue. 360 clusters were searched for possible members, excluding nearby clusters with distances $D<200$ pc. Members were selected using ground based information (photometry, radial velocity, proper motion, distance from the cluster centre) and information provided by Hipparcos (proper motion, parallax). Altogether 630 certain and 100 possible members were found. A comparison of the Hipparcos parallaxes with photometric distances of open clusters shows good agreement. The Hipparcos data confirm or reject the membership of several Cepheids in the studied clusters.
\end{abstract}

Key words: Galaxy: open clusters and associations: general — Astrometry — stars: variables: Cepheids

\section{Introduction}

The aim of this work is to determine proper motions and parallaxes of open clusters using the Hipparcos Catalogue (ESA 1997). We will focus here on the more distant open clusters and try to find proper motions for all clusters with distances greater then $D=200 \mathrm{pc}$. In combination with accurate photometric distances and radial velocities, the proper motions will provide valuable information on the kinematic parameters of the galactic rotation curve and the distance to the galactic centre. They are also useful to study the formation and evolution of the open cluster

Send offprint requests to: H. Baumgardt

* Tables 1 and 2 are only available in electronic form at the CDS via anonymous ftp to cdsarc.u-strasbg.fr (130.79.128.5) or via http://cdsweb.u-strasbg.fr/Abstract.html system. These questions will be addressed in a forthcoming paper. The present paper provides the observational database for these studies.

Stars in open clusters were searched and proposed by Wielen \& Dettbarn (1985) and selected by a specific working group within the INCA Consortium during the creation of the Hipparcos Input Catalogue. However, the Hipparcos Catalogue may contain additional cluster stars not selected by this working group: first, there may exist bright cluster stars that have so far not been studied at all or were studied in detail only after the creation of the Hipparcos Input Catalogue. Such stars could have entered the Hipparcos Catalogue as part of the all sky survey. In addition, cluster stars may have been proposed by other research groups if they are of astrophysical interest.

It therefore seemed appropriate to perform a new search for stars in open clusters. Sections 2 and 3 describe the selection of the open clusters and the search for Hipparcos stars in the cluster fields.

Section 4 describes the way in which cluster members were identified among the candidates and the next section describes how the mean astrometric parameters of the clusters were determined. Section 6 lists the classification of the Hipparcos stars and presents the mean astrometric parameters of the open clusters. Two applications of the astrometric parameters are also described in this section. First, we compare the Hipparcos parallaxes with the photometric parallaxes of the open clusters to check for systematic errors in both methods. Second we use the proper motions of the open clusters to check the membership of several Cepheids. Section 7 summarises our results.

\section{Cluster selection}

The basic data of the open clusters (positions, distances, magnitudes of brightest stars) were taken from the catalogue of Lyngå (1987). 1151 open clusters are listed 
in his catalogue, most of which however are too faint for Hipparcos. We therefore removed all clusters that have never been studied at all, since they are presumably very faint and even if they contain stars bright enough for Hipparcos, there is no information available to identify them as members. In addition, we omitted clusters where, according to Lyngå (1987), brightest members are fainter than $V=12 \mathrm{mag}$, since it is very unlikely that they contain Hipparcos stars. Nearby clusters with distances $D<200$ pc were also omitted from our analysis. This was done because they have large angular diameters, so that the assumption of a common proper motion of all cluster stars is not valid for them. They require a different kind of analysis (for example convergent point methods like in Perryman et al. 1998), which is beyond the scope of the present paper. Nearby clusters not discussed here include the Hyades, Pleiades, Coma Ber, IC 2391, IC 2602, $\alpha$ Persei Cluster, Praesepe (NGC 2632) and the UMA Star Cluster. Hipparcos results for most of them can already be found in the literature (Perryman et al. 1998; van Leeuwen 1999; Robichon et al. 1999). Our final list contained 360 clusters which may have members in the Hipparcos Catalogue.

We note that Lyngå (1987) lists two additional clusters with distances less than 200 pc (Col 399, Upg 1), but Baumgardt (1998) has recently shown on the basis of proper motions from the Hipparcos and ACT (Urban et al. 1997) catalogues that they do not exist at all. We finally note that Platais et al. (1998) found several new cluster candidates in the Hipparcos Catalogue, but most of them require further study to confirm their reality.

\section{Member search}

Stars can only be gravitationally bound to a star cluster if they are located inside its tidal radius. If a star cluster is seen from outside, this corresponds to a spherical region on the sky inside which stars must lie in order to be bound to the cluster. To find the members, we therefore took a rough estimate for the tidal radius (we assumed $12 \mathrm{pc}$, the tidal radius for a $1000 M_{\odot}$ star cluster in the solar neighbourhood), the cluster distance from Lyngå (1987), and calculated the angular diameters of these regions. They were then searched for Hipparcos stars and 2900 stars were found altogether.

\section{Member selection}

The crucial part in the determination of the mean astrometric parameters of star clusters is the proper separation of members and non-members. This is especially important in the present case since we will typically have only a few stars per cluster (sometimes only one star), so that a single misclassified field star can already influence our final solution considerably. Since the information provided by Hipparcos is not sufficient to separate the cluster members from the field stars, we had to judge the membership by combining Hipparcos data with information provided by ground based studies. These include:

- Photometry and spectroscopy;

- Proper motions;

- Radial velocities;

- Parallaxes;

- Angular distances from the cluster centres.

The following paragraphs will illustrate this approach.

\subsection{Photometry and spectroscopy}

Multicolour photometry and spectroscopy were our main criteria for the membership determination due to the fact that they are available for most stars and rule out membership for many of them. We performed a literature search for each cluster and noted the classification of our candidates in the various studies. We did not examine the membership on our own, since in most photometric studies the data was already carefully analysed by the authors themselves.

\subsection{Proper motions}

The main proper motion source is the Hipparcos Catalogue itself. If the proper motion of a cluster is already known from a few certain members in the Hipparcos Catalogue, it is possible to check the membership of the remaining stars with the proper motion of the cluster: new members are expected to have proper motions in agreement with the known members. However, the majority of our clusters do not have proper motions which clearly separate them from the field stars, therefore a matching proper motion in the Hipparcos Catalogue is a necessary but not a sufficient criterion for cluster membership.

In some cases we combined the Hipparcos proper motions with proper motions of additional members found in the TRC Catalogue (Høg et al. 1998). This was done for clusters where we could find no common motion among the Hipparcos stars or where only one member could be found in Hipparcos and we regarded it necessary to check its membership further. We also checked our proper motions against proper motions derived by Glushkova et al. (1999) from the TRC Catalogue.

Ground based proper motions were also used for the member determination. They are available for about 40 of the studied clusters. Ground based proper motions have generally the same or an even higher accuracy than the Hipparcos proper motions. Unfortunately, since they are not on the Hipparcos system, they cannot be directly compared with Hipparcos. Due to their accuracy, they were 
nevertheless a powerful tool to eliminate field stars from our sample.

\subsection{Radial velocities}

High precision radial velocities are best suited to distinguish between members and non-members. Since they are available for relatively few stars only, their application is limited to a small number of clusters. They nevertheless give valuable information, since the studied stars are often giants or variable stars for which photometry and spectroscopy are of limited use.

\subsection{Parallaxes}

Parallaxes are provided by the Hipparcos satellite. They put tight limits to the distances of nearby stars, but get less and less accurate the further the star is away. For most clusters they were able to eliminate a few foreground stars, but the majority of the field stars have parallaxes which would be compatible with the assumption of a cluster membership.

\subsection{Angular distance from the cluster centre}

While the surface density of a star cluster drops from the inner to the outer parts, the density of the background remains essentially constant. Thus, a star seen close to the cluster centre is more likely a member than a star near the tidal radius. The angular distance therefore gives some hints for the classification of stars.

Our final classification was made by taking into account all the information we could get. Stars were divided into three categories (members, possible members and non-members) according on how well they fulfilled the membership criteria.

\section{Determination of the mean astrometric parameters}

We neglected perspective effects and assumed that all cluster stars have similar proper motions and parallaxes. This is justified by the large distances and resulting small angular diameters of the clusters studied. Due to the large distances the internal motions of the cluster stars can also be neglected.

Stars included in the Double and Multiple Systems Annex of the Hipparcos Catalogue, i.e. with C, G, V, O or $\mathrm{X}$ entries in field $\mathrm{H} 59$, were not used to derive the mean astrometric parameters, since the astrometric solution derived by Hipparcos is affected by the binary nature of these stars, as described in Robichon et al. (1999).
The mean astrometric parameters were kindly calculated for us by Floor van Leeuwen. For clusters containing more than one star, he used the method described in van Leeuwen \& Evans (1998) to calculate the mean astrometric parameters. This method takes into account the correlations that exist between the abscissae residuals of Hipparcos stars measured on the same reference great circles. These correlations increase with decreasing angular separation and are important in our case due to the small angular diameters of the clusters studied. No such correlations exist for clusters containing only one star, so we took the solution for these clusters directly from the solution given for the single member in the Hipparcos Catalogue.

Alternatively, we also calculated the astrometric parameters without taking small-scale correlations into account. This was done to estimate the influence of these correlations. For that purpose, the cluster solutions were obtained from the member stars by taking the mean of their Hipparcos solutions.

\section{Results}

For 140 clusters no members could be found in the Hipparcos Catalogue. In the remaining 205 clusters, we found altogether 630 certain and 130 possible members. They are listed in Table 1 together with some non-members. The majority of non-members were never supposed to be cluster stars since they have colours, magnitudes and angular distances that clearly rule out their membership. In order to keep Table 1 short, we therefore list only non-members which could be found in the "Database for Galactic Open Clusters" (BDA) by Mermilliod (1995). These stars are presumably much closer to the cluster centre than the majority of nonmembers and many of them were previously thought to be members.

Column 8 of Table 1 gives a membership probability derived from the proper motions. It was calculated as follows: If a star was not used to calculate the mean cluster motion, we took the difference $\boldsymbol{x}$ between the proper motion of the cluster (taken from Table 2) and the star and calculated the product of this difference with the sum $\Sigma$ of the covariance matrices of the star and the cluster according to:

$c=x^{\prime} \Sigma^{-1} x$.

The dimensionless number $c$ was then transformed into a membership probability under the assumption that it is distributed like a chi-square distribution with two degrees of freedom. If a star was used for the derivation of the mean cluster motion, we first calculated a solution for the cluster without using the star in question and compared the proper motion of the star with this new solution.

Most non-members were classified as such due to their large proper motion differences relative to the mean cluster motion. The remaining stars that were classified 


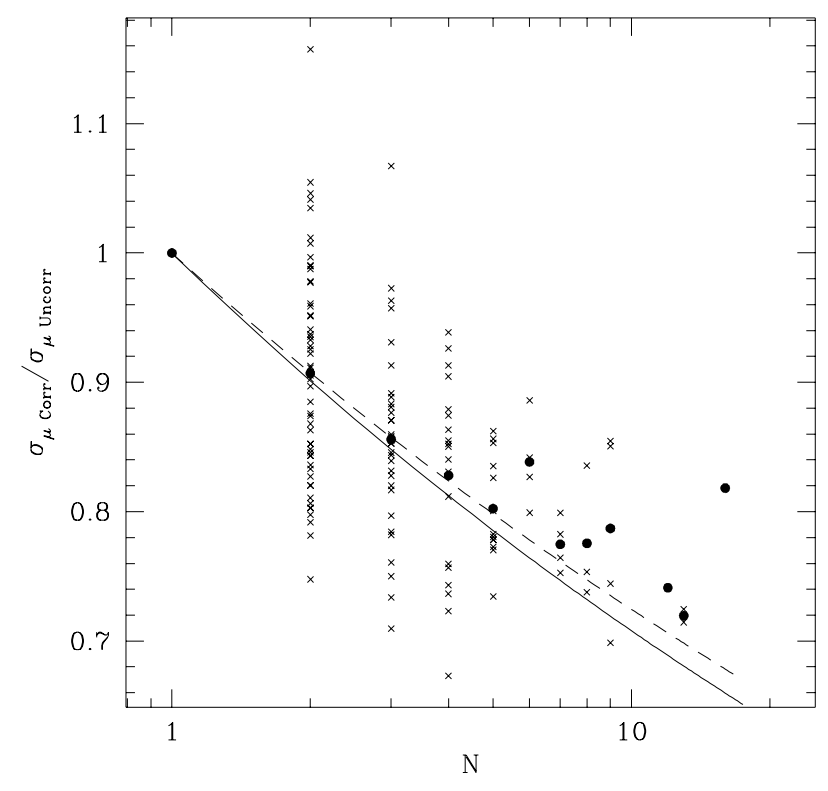

Fig. 1. Proper motion errors derived by taking small-scale correlations into account compared to the uncorrelated solution as a function of the number of cluster stars $N$. The crosses show individual clusters, the dots mark the mean values for a given $N$. The solid line shows a dependence proportional to $N^{-0.15}$ (exponent derived from Lindegren 1988), the dashed line shows one proportional to $N^{-0.14}$

as non-members were either too far away from the cluster centre to be bound or had a photometry which was incompatible with the assumption of cluster membership. Possible members with a high probability for membership are often not well-studied, outlying stars. They may be members according to their proper motion and photometry.

Table 2 presents our final solution for the astrometric cluster parameters, determined as outlined in Sect. 5. The unit weight standard deviations show some scatter around the theoretical value of one. This is caused by the small number of stars available for most clusters. The mean over all clusters that contain at least two stars is 1.01, which is very close to the theoretical value. We note that the unit weight standard deviations are generally smaller than one for clusters with more than 5 stars in the astrometric parameter determination. Since the correlations of the abscissae residuals become more important for clusters containing many stars, this may indicate that these correlations were slightly overestimated when the mean values were calculated. If the small-scale correlations are not taken into account, we obtain a mean unit weight standard deviation of 0.97 for all clusters, which is also very close to the theoretical value of one.

Figure 1 compares the errors obtained by taking smallscale correlations into account with the errors of the uncorrelated solution. Shown is the ratio of the sums $\sigma_{\mu}$ of the proper motion errors $\sigma_{\mu}=\sqrt{\sigma_{\mu \alpha *}^{2}+\sigma_{\mu \delta}^{2}}$ as

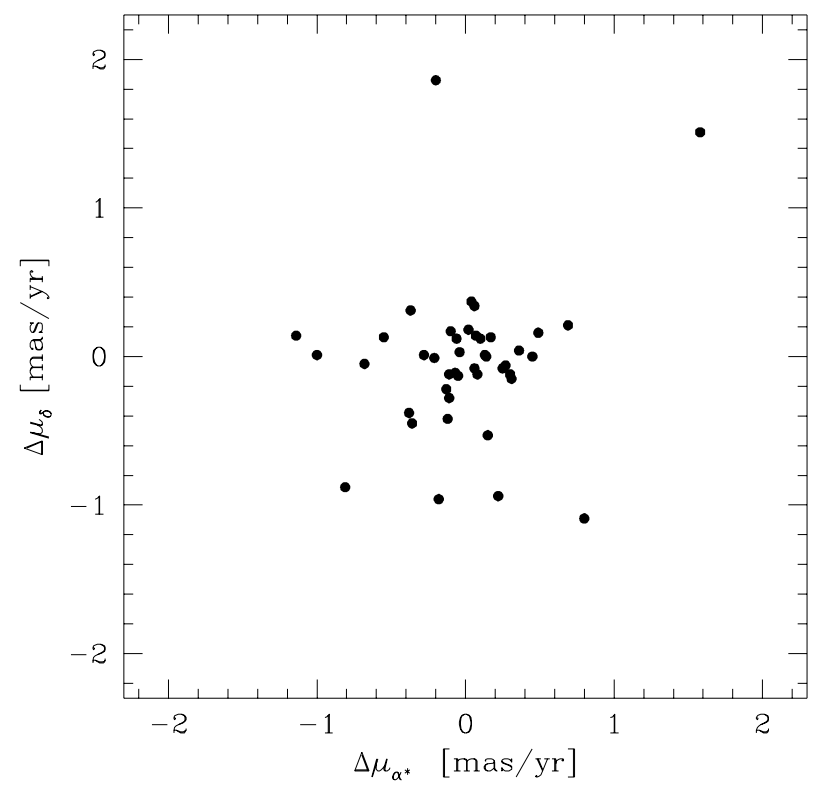

Fig. 2. Plot of the proper motions derived in this work minus the proper motions from Robichon et al. (1999) for the clusters in common

a function of the number of cluster stars $N$. If a cluster contains only one star, this ratio must be unity, since both solutions are taken directly from Hipparcos. For clusters with more than one star, the mean error should be proportional to $N^{-0.5}$ in the uncorrelated case. For the correlated case, Lindegren (1988) estimated an $N^{-0.35}$ dependence, so the ratio of the errors should be proportional to $N^{-0.15}$. Clusters containing less than 6 Hipparcos stars are best fitted by a $N^{-0.14}$ law, very close to the expected value. An $N^{-0.36}$ decrease can therefore be taken as a rule of thumb to estimate the errors of the astrometric parameters for correlated measurements in Hipparcos.

Robichon et al. (1999) determined absolute proper motions and parallaxes of all clusters that are closer than $300 \mathrm{pc}$ or have more than 4 members in the Hipparcos Catalogue. Figure 2 compares our proper motions with theirs for the clusters in common. Although they also use the Hipparcos Catalogue and a similar method to derive the proper motions, the final results differ by typically $0.5 \mathrm{mas} / \mathrm{yr}$, which is of the same order as the quoted errors. The differences are due to differences in the stars selected as cluster members and slight differences in the abscissae formal errors and correlations that are used in the methods of Robichon et al. (1999) and van Leeuwen \& Evans (1998).

\subsection{Comparison of the Hipparcos parallaxes with photometric distances}

Loktin et al. (1994, 1997) and Dambis (1999) have determined distances and ages of open clusters on the basis of published photometry. Their data represent large and 
homogeneous parameter sets. With the help of the Hipparcos parallaxes, we can check for global errors $f$ in their distance scales:

$R_{\text {Phot }}=f \cdot R_{\text {true }}$.

Such errors can be detected with a test similar to that used by Feast \& Catchpole (1997): the (true) distance of a cluster is connected with the cluster parallax $\pi$ through the equation

$\pi=1 / R_{\text {true }}$,

so that an estimate for $f$ can be obtained by inserting Eq. (3) into (2):

$f=0.001 \pi_{\text {Hip }} R_{\text {Phot }}$.

Here the Hipparcos parallax $\pi_{\text {Hip }}$ is measured in mas and the photometric distance is in parsecs. The mean over all clusters was taken to derive $f$ :

$<f>=\frac{\sum \frac{f_{i}}{\sigma_{i}^{2}}}{\sum \frac{1}{\sigma_{i}^{2}}}$.

The errors $\sigma_{i}$ on the right-hand side are a combination of the errors in the Hipparcos parallaxes and the (random) errors in the photometric distances to individual clusters, taken to be $10 \%$ for the Loktin sample. Since the error estimate of the photometric distance is done with the observed distance and not with the true one, the above test leads to a slightly biased estimate for $f$. Monte-Carlo simulations show that this bias remains small as long as the errors in the photometric distances are not of the order of $40 \%$ or larger.

From 186 clusters in common between Loktin et al. (1997) and this work, we derive a correction of $f=$ $1.12 \pm 0.05$ to their distance scale, i.e. their distances should be decreased by $12 \%$. Part of this decrease may be explained by the fact that Loktin et al. assumed an Hyades distance modulus of $(M-m)_{0}=3.42$, which is too large since the Hipparcos data indicate $(M-m)_{0}=3.33$ (Perryman et al. 1998). The errors in the photometric distances of Dambis (1999) are taken from their work. From 117 clusters in common, we obtain a correction factor of $f=0.99 \pm 0.06$. Their overall distance scale is therefore in very good agreement with Hipparcos.

Using the test of Arenou \& Luri (1999), we can also check the parallax errors in the Hipparcos Catalogue: the difference $\Delta \pi$ between the Hipparcos parallax and the photometric parallax $\pi_{\text {Phot }}=1 / R_{\text {Phot }}$

$\Delta \pi=\pi_{\text {Hip }}-\pi_{\text {Phot }}$

is mainly caused by the error in the Hipparcos parallax, since the errors in the photometric parallaxes are only of the order of 0.1 mas. If the errors in the Hipparcos Catalogue are normally distributed and show no correlations other than those that were already accounted for in Sect. 5 , the ratio of $\Delta \pi / \sigma_{\text {Hip }}$ should also be normally distributed.

Figure 3 shows the distribution of $\Delta \pi / \sigma_{\text {Hip }}$ for the Loktin et al. distances. A normal distribution provides

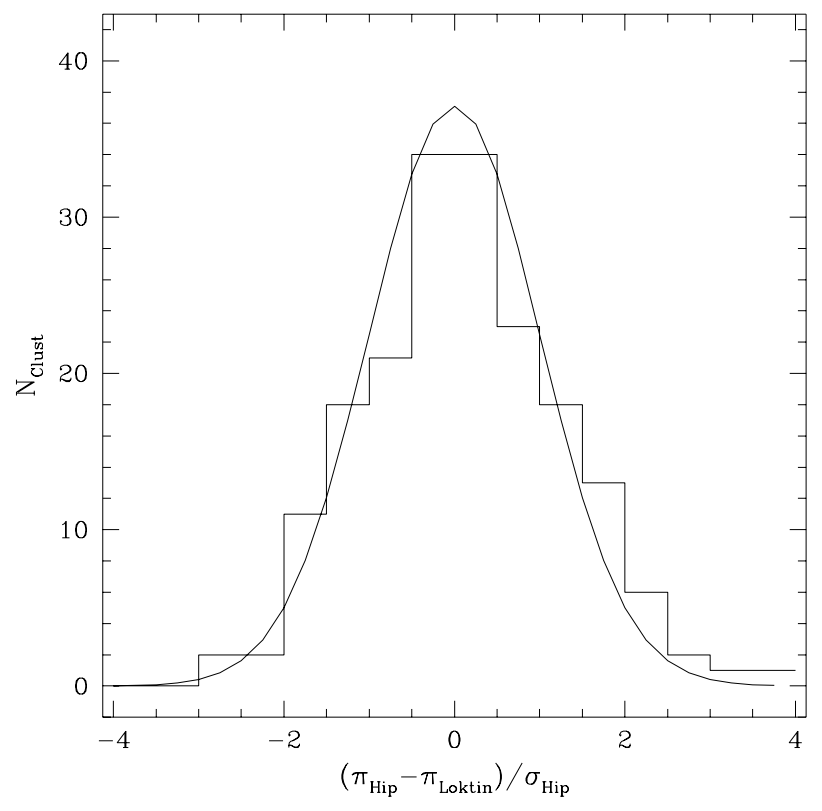

Fig. 3. Histogram of the normalised differences $\left(\pi_{\mathrm{Hip}}-\right.$ $\left.\pi_{\text {Loktin }}\right) / \sigma_{\text {Hip }}$. A Gaussian provides a very good fit to the data. Correlated errors of the order of 0.5 mas or larger would significantly broaden the observed distribution and can therefore be ruled out

a very good fit to the data. Narayanan \& Gould (1999) proposed correlated errors extending over angular scales of 2 to 3 degrees and with amplitudes of up to 2 mas in the Hipparcos parallaxes as the reason for the discrepancy between recent photometric distances and the Hipparcos distance to the Pleiades. Our clusters have small angular sizes and would be effected by such errors as a whole. If they exist in the entire Hipparcos Catalogue, such errors would significantly broaden the cluster distribution in Fig. 3 (note that typical parallax errors of our clusters are only 0.5 mas). Since such a broadening is not observed we can rule out correlated errors with amplitudes of more than a few tenths of a mas for the vast majority of our clusters. A similar conclusion was drawn by Arenou \& Luri (1999). We confirm their results with a larger database. We finally note that a similar result is obtained if the distances of Dambis (1999) are used.

\subsection{Cepheids in open clusters}

The Hipparcos proper motions (and to a lesser degree also the parallaxes) confirm or reject the cluster membership of stars which are of astrophysical interest, like e.g. WolfRayet stars, red giants and various types of variable stars. This may help to better define their physical parameters. As an example, we discuss the membership of Cepheids in our clusters.

11 Cepheids are included in Table 1. Many of them are the only Hipparcos star in their cluster, so our classification is entirely based on results found in the literature. 
Eight Cepheids are in clusters with two or more members (see Table 3 and Fig. 4).

The membership of U Sgr (HIP 90836) in IC 4725 and of S Nor (HIP 79932) in NGC 6087 was already discussed by Lyngå \& Lindegren (1998) on the basis of the Hipparcos data. We confirm the cluster membership of both Cepheids. DL Cas (HIP 2347) is a highly probable member of NGC 129 on the basis of its photometry (Turner et al. 1992), radial velocity (Mermilliod et al. 1987) and proper motion (Lenham \& Franz 1961). The Hipparcos data confirm these results: from two cluster members in the Hipparcos Catalogue we derive a cluster motion which gives a membership probability of $34 \%$ for DL Cas. The Cepheid is therefore very likely a cluster member.

V Cen (HIP 71116) is a member of NGC 5662 according to Claria et al. (1991). Figure 4a shows the proper motions of the Cepheid and the other cluster stars. The agreement is excellent and the Cepheid has a high membership probability of $90 \%$. We conclude that it is a member of NGC 5662.

The membership of SZ Tau (HIP 21517) in NGC 1647 was proposed by Efremov (1964a, 1964b) and confirmed by Turner (1992) on the basis of $U B V$ photometry and spectroscopy. Geffert et al. (1996), based on proper motions from photographic plates, denied the cluster membership of the Cepheid. We could find five members of NGC 1647 (three certain, two possible) in the Hipparcos Catalogue. Their mean proper motion differs significantly from the motion of the Cepheid and rules out its membership. Despite a rough agreement in the radial velocities of the cluster and the Cepheid (see the discussion in Turner 1992), we conclude that SZ Tau is not a member of NGC 1647.

EV Sct (HIP 91239) and Y Sct (HIP 91366) are possible members of NGC 6664. The membership of EV Sct is well established by its radial velocity (Mermilliod et al. 1987). The membership of $Y$ Sct is also very likely since its mean radial velocity of $\gamma=17.8 \mathrm{~km} \mathrm{~s}^{-1}$ (Moffett \& Barnes 1987) is in good agreement to the mean cluster velocity of $r_{\mathrm{v}}=17.8 \pm 0.2 \mathrm{~km} \mathrm{~s}^{-1}$ given by Mermilliod et al. (1987). The Hipparcos data is in agreement with the radial velocities, since both Cepheids have high membership probabilities of $81.8 \%$. The cluster motion is however not very well established since the two Cepheids are the only cluster members in the Hipparcos Catalogue.

The situation is less clear for the Cepheids BB Sgr (HIP 92491) and GH Car (HIP 54621). BB Sgr was proposed to be a coronal member of Col 394 by Tsarevsky et al. (1966). Its membership was later confirmed by Turner \& Pedreros (1985) on the basis of $U B V R I$ photometry and by Gieren et al. (1998) on the basis of new calibrations of the surface brightness (Barnes-Evans) method. The Hipparcos data are inconclusive. Two Hipparcos members give a low membership probability of $2.7 \%$ for BB Sgr, which is not completely
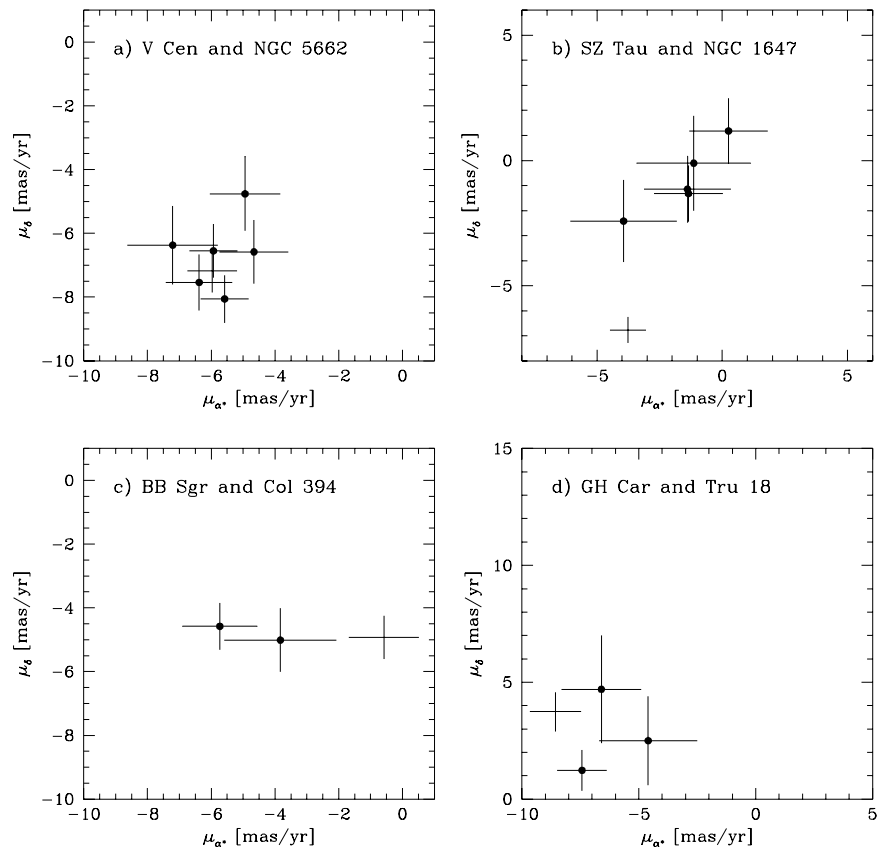

Fig. 4. Proper motions of Cepheids in clusters with at least two other Hipparcos members, see Table 4. Not shown are U Sgr in IC 4725 and S Nor in NGC 6087, which were already discussed by Lyngå \& Lindegren (1998), and EV Sct and Y Sct in NGC 6664. Cluster stars are shown by filled circles, Cepheids without symbols

inconsistent with the assumption of a cluster membership. If additional members from the TRC Catalogue are taken into account, the membership probability of BB Sgr drops to below $0.1 \%$, raising serious doubts on its cluster membership. We note here that the relative proper motion of the Cepheid is pointing towards the cluster, which advocates against a common origin of both. Given also the large angular distance from the cluster centre, we conclude that BB Sgr is unlikely to be a member of Col 394.

GH Car is a member of Tru 18 according to Vazquez $\&$ Feinstein (1990). From three stars in the Hipparcos and TRC catalogues (1 from Hipparcos, 2 from the TRC Catalogue), we derive a mean proper motion of $\left(\mu_{\alpha *} / \mu_{\delta}\right)=(-6.79 \pm 0.83 / 1.79 \pm 0.75) \mathrm{mas} / \mathrm{yr}$ for the cluster. This gives a relatively low membership probability of $10 \%$ for GH Car. However, there is a discrepancy in the photometric distances: from their $U B V R I$-photometry, Vazquez \& Feinstein (1990) found a cluster distance of $D=1550 \mathrm{pc}$. The Cepheid seems to be located further away, since the PL-relation of Feast \& Catchpole (1997) gives an absolute magnitude of $M_{V}=-3.55$ and a distance of $D=2313 \mathrm{pc}$ assuming that the period, mean $V$ magnitude and reddening of $\mathrm{GH}$ Car are given by $P=5.72557 \mathrm{~d}$, $\langle V\rangle=9.17 \mathrm{mag}$ and $E(B-V)=0.29 \mathrm{mag}$ (Vazquez $\&$ Feinstein 1990). GH Car is probably not physically 
Table 3. Proper motions of Cepheids and open clusters. The proper motions of the clusters are calculated without taking the motions of the Cepheids into account. The constants $c$ were calculated according to Eq. (1) and the membership probabilities in Col. 12 were derived under the assumption that $c$ is distributed like a chi-square distribution with two degrees of freedom

\begin{tabular}{|c|c|c|c|c|c|c|c|c|c|c|c|c|}
\hline \multirow{3}{*}{$\begin{array}{l}\text { Cepheid } \\
\text { U Sgr }\end{array}$} & \multirow{3}{*}{$\begin{array}{l}\text { Cluster } \\
\text { IC } 4725\end{array}$} & \multirow{2}{*}{\multicolumn{4}{|c|}{\begin{tabular}{cc|cc}
\multicolumn{3}{c}{ Proper motion Cepheid } \\
$\mu_{\alpha *}$ & $\sigma_{\mu}$ & $\mu_{\delta}$ & $\sigma_{\mu}$ \\
{$[\mathrm{mas}$} & $/ \mathrm{yr}]$ & & {$[\mathrm{mas} / \mathrm{yr}]$} \\
\end{tabular}}} & \multicolumn{4}{|c|}{ Proper motion Cluster } & \multirow{3}{*}{$\begin{array}{c}c \\
3.83\end{array}$} & \multirow{3}{*}{$\begin{array}{r}\text { Mem. } \\
\text { Prob. } \\
14.7\end{array}$} & \multirow{3}{*}{$\begin{array}{l}\text { Hipparcos stars used to calculate } \\
\text { the proper motion of the cluster } \\
90801,90900\end{array}$} \\
\hline & & & & & & \multicolumn{2}{|c|}{$\begin{array}{cc}\mu_{\alpha *} & \sigma_{\mu} \\
{[\text { mas }} & / \mathrm{yr}] \\
\end{array}$} & \multicolumn{2}{|c|}{$\begin{array}{lr}\mu_{\delta} & \sigma_{\mu} \\
{[\text { mas }} & / \mathrm{yr}] \\
\end{array}$} & & & \\
\hline & & -4.15 & 0.94 & -6.05 & 0.70 & -1.68 & 0.87 & -6.39 & 0.62 & & & \\
\hline S Nor & NGC 6087 & -1.10 & 0.77 & -1.20 & 0.70 & -1.67 & 0.71 & -1.60 & 0.66 & 0.51 & 77.3 & $79891,79907,79973$ \\
\hline DL Cas & NGC 129 & -0.75 & 1.05 & -1.38 & 0.73 & -2.67 & 0.94 & -1.85 & 0.65 & 2.16 & 33.9 & 2354,2382 \\
\hline V Cen & NGC 5662 & -5.97 & 0.78 & -7.18 & 0.67 & -5.60 & 0.50 & -7.33 & 0.51 & 0.21 & 90.0 & $\begin{array}{l}\text { 71163, 71326, 71334, 71378, 71397, } \\
71398\end{array}$ \\
\hline SZ Tau & NGC 1647 & -3.76 & 0.72 & -6.77 & 0.52 & -1.37 & 0.97 & -1.02 & 0.77 & 41.6 & 0.0 & $21875,22112,22161,22185,22211$ \\
\hline EV Sct & NGC 6664 & 1.16 & 2.31 & -2.84 & 1.73 & -0.63 & 1.65 & -2.20 & 1.29 & 0.49 & 81.8 & 91366 \\
\hline Y Sct & NGC 6664 & -0.63 & 1.65 & -2.20 & 1.29 & 1.16 & 2.31 & -2.84 & 1.73 & 0.49 & 81.8 & 91239 \\
\hline BB Sgr & Col 394 & -0.58 & 1.10 & -4.93 & 0.68 & -4.80 & 1.12 & -4.78 & 0.69 & 7.23 & 2.7 & 92505,92650 \\
\hline BB Sgr & Col 394 & -0.58 & 1.10 & -4.93 & 0.68 & -4.79 & 0.44 & -6.22 & 0.37 & 15.43 & 0.0 & $\begin{array}{l}92505,92650,1,6,12,22,26,27,28,33 \\
52,53,55,57,58,59,60,62,63,66,67,76\end{array}$ \\
\hline GH Car & Tru 18 & -8.56 & 1.08 & 3.74 & 0.84 & -6.79 & 0.83 & 1.79 & 0.75 & 4.68 & 9.6 & $54668,11,16$ \\
\hline
\end{tabular}

Notes: Col 394: The numbers of the TRC stars in Col. 13 are taken from Claria et al. (1991). Tru 18: The numbers of the two TRC stars are from Vazquez \& Feinstein (1990).

related to Tru 18. Radial velocities would help to confirm our conclusions concerning the last two Cepheids.

\section{Summary}

The proper motions and parallaxes of 205 open clusters were determined from 630 certain and 100 possible members found in the Hipparcos Catalogue. A comparison of the parallaxes with photometric distances from Loktin et al. (1997) argues for a decrease of the photometric distance scale by $12 \% \pm 6 \%$, while the distance scale of Dambis (1999) is in good agreement with Hipparcos. No evidence for unaccounted small-scale correlated errors in the Hipparcos Catalogue is found by these comparisons. It therefore seems unlikely that such errors can explain the discrepancy between the Hipparcos parallax and photometric distance estimates of the Pleiades as proposed by Narayanan \& Gould (1999).

With the help of the Hipparcos proper motions, we can confirm the membership of the Cepheids U Sgr in IC 4725, S Nor in NGC 6087, DL Cas in NGC 129 and $\mathrm{V}$ Cen in NGC 5662 and can reject the membership of SZ Tau in NGC 1647. The improved membership information may lead to better estimates for the absolute magnitudes of Cepheids.

Acknowledgements. We are grateful to Floor van Leeuwen for calculating the correlated solutions of the astrometric parameters of the open clusters for us. We acknowledge the help of Elena Glushkova and Alexander Loktin who send us their data prior to publication. We also thank Sabine Frink and an anonymous referee for making suggestions which improved the paper. Extensive use has been made of the Simbad and BDA databases. HB is supported by the Sonderforschungsbereich 328 Entwicklung von Galaxien.

\section{References}

Amieux G., Burnage R., 1981, A\&AS 44, 101

Arenou F., Luri X., 1999, in Harmonizing Cosmic Distance Scales in a Post-Hipparcos Era, Egret D., Heck A. (eds.), ASP Conf. Ser. 167, 13

Balona L.A., Laney C.D., 1995, MNRAS 277, 250

Baumgardt H., 1998, A\&A 340, 402

Becker W., 1963, ZA 57, 128

Claria J.J., 1977a, PASP 89, 803

Claria J.J., 1977b, A\&AS 27, 145

Claria J.J., Lapasset E., Bosio M.A., 1991, MNRAS 249, 193

Dambis A., 1999, Astron. Lett. 25, 10

Eggen O.J., 1983, AJ 88, 197

Efremov Y.N., 1964a, Peremennye Zvezdy 15, 242

Efremov Y.N., 1964b, Astron. Tsirk. 292, 3

ESA, 1997, The Hipparcos and Tycho Catalogues, SP-1200

Feast M.W., Catchpole R.M., 1997, MNRAS 286, L1

Feinstein A., Vázquez R.A., 1989, A\&AS 77, 321

Fitzgerald M.P., Moffat A.F.J., 1975, A\&AS 20, 289

Forte J.C., 1976, A\&AS 25, 271

Geffert M., et al., 1996, A\&AS 118, 277

Gieren W.P., Fouqué P., Gómez M., 1998, ApJ 496, 17

Glushkova E.V., 1999 (private communication)

Glushkova E.V., Rastorguev A.S., 1991, SvAL 17, 13

Havlen R.J., 1976, A\&A 50, 227

Hobbs R.W., 1961, AJ 66, 517

Høg E., et al., 1998, A\&A 335, 65

Jenkner H., Maitzen H.M., 1987, A\&AS 71, 255

Johnson H.L., 1961, Low. OB 5, 133

King D.S., 1981, Syd. Obs. Papers 89

Lee P.D., Perry C.L., 1971, AJ 76, 464 
van Leeuwen F., 1999, A\&A 341, L71

van Leeuwen F., Evans D.W., 1998, A\&AS 130, 157

Lenham A.P., Franz O.G., 1961, AJ 66, 16

Lindegren L., 1988, in Proceedings of a colloquium on the European Astrometry Satellite Hipparcos - Scientific aspects of the Input Catalogue Preparation II, Torra J., Turon C., Blaauw A. (eds.), p. 179

Lindoff U., 1968, Ark. Astron. 5, 63

Lindoff U., 1973, A\&AS 9, 229

Liu T., Janes K.A., Bania T.M., 1991, AJ 102, 1103

Loden L.O., 1965, ApJ 141, 668

Loden L.O., 1966, Ark. Astron. 4, N5

Loktin A., Matkin N., 1994, Astron. Astrophys. Trans. 4, 153

Loktin A., Zakharova P., Gerasimenko T., Malisheva L., 1997, Balt. Ast. 6, 316

Lyngå G., 1987, Lund Catalogue of Open Cluster Data, 5th ed., Stellar Data Centre, Observatoire de Strasbourg, France

Lyngå G., Lindegren L., 1998, NewA 3, 121

Maitzen H.M., Seggewiss W., Tueg H., 1981, A\&A 96, 174

McNamara B.J., Hack W.J., Olson R.W., Mathieu R.D., 1989, AJ 97,1427

Mermilliod J.-C., Mayor M., Burki G., 1987, A\&AS 70, 389

Mermilliod J.-C., 1995, in Information and On-Line Data in Astronomy, Egret D., Albrecht M.A. (eds.). Kluwer Academic Press, Dordrecht, p. 127

Moffat A.F.J., Vogt N., 1973, A\&AS 10, 135

Moffat A.F.J., Vogt N., 1975, A\&AS 20, 125

Moffett T.J., Barnes III T.G., 1987, PASP 99, 1206

Narayanan V.K., Gould A., 1999, ApJ 523, 328

Peña J.H., Peniche R., 1994, RMxAA 29, 125

Perry C.L., 1973 in Spectral Classification and Multicolour
Photometry, IAU Symposium 50, Fehrenbach C., Westerlund B.E. (eds.), p. 192

Perry C.L., Hill G., Christodoulou D.M., 1991, A\&AS 90, 195

Perryman M.A.C., et al., 1998, A\&A 331, 81

Platais I., Kozhurina-Platais V., Barnes S., Horch E.P., 1996, BAAS 28, 822

Platais I., Kozhurina-Platais V., van Leeuwen F., 1998, AJ 116, 2423

Prosser C.F., 1993, AJ 105, 1441

Robichon N., Arenou F., Mermilliod J.-C., Turon C., 1999, A\&A 345, 471

Röser S., Bastian U., 1994, A\&A 285, 875

Ruciński S.M., 1987, PASP 99, 487

Schmidt-Kaler T., 1961, ZA 53, 1

Sher D., 1965, MNRAS 129, 237

Stone R.C., 1977, A\&A 54, 803

Svolopoulos S.N., 1961, ApJ 134, 612

Tsarevsky G.S., Ureche V., Efremov Y.N., 1966, Astron. Tsirk. 367,1

Turner D.G., 1992, AJ 104, 1865

Turner D.G., 1976, AJ 81, 1125

Turner D.G., Forbes D., Pedreros M., 1992, AJ 104, 1132

Turner D.G., Pedreros M., 1985, AJ 90, 1231

Urban S.E., Corbin T.E., Wycoff G.L., 1997, U.S. Naval Observatory, Washington DC

Vazquez R.A., Feinstein A., 1990, A\&AS 86, 209

Williams P.M., 1978, MNRAS 183, 49

Wielen R., Dettbarn C., 1985, in Colloquium on The European Astrometry Satellite HIPPARCOS. Scientific Aspects of the Input Catalogue preparation., Guyenne T.D., Hunt J. (eds.), p. 145 